\title{
A NONLINEAR ELLIPTIC BOUNDARY VALUE PROBLEM RELATED TO CORROSION MODELING
}

\author{
BY \\ MICHAEL VOGELIUS AND JIAN-MING XU \\ Department of Mathematics, Rutgers University, New Brunswick, NJ and \\ Département de Mathématiques, École Polytechnique Fédérale de Lausanne, Switzerland
}

\begin{abstract}
We study a nonlinear boundary value problem arising from electrochemistry. The essential difficulties are due to the strong nonlinear nature of part of the boundary condition. This part of the boundary condition is of an exponential type and is normally in the corrosion literature associated with the names of Butler and Volmer. We examine the questions of existence and uniqueness of solutions to this boundary value problem. In a numerical example we compare the behaviour of the solutions to the nonlinear problem with the behaviour of the solutions to a corresponding linearized problem. In contrast to earlier studies we put a major emphasis on studying parameter values that may be relevant for the case in which part of the boundary is in a transition to passivity - in practice most likely because it is nearly covered by an oxide layer.
\end{abstract}

0. Introduction. In this paper we shall study a nonlinear boundary value problem arising from galvanic corrosion. Before proceeding to a mathematical analysis of this problem we shall devote this first section to a brief explanation of some background material. Consider the battery cell illustrated in Fig. 1 (see p. 480). It consists of two strips of metal (one of silver and one of zinc) partially emerged in a saltwater solution. It is well known that chemical reactions at each metal strip will result in a current passing between the two. At the silver strip, hydrogen gas is produced,

$$
2 \mathrm{H}_{2} \mathrm{O}+2 e^{-} \rightarrow \mathrm{H}_{2} \uparrow+2 \mathrm{OH}^{-} \text {. }
$$

Here the reactant gains electrons, i.e., one says that a reduction takes place, and the strip is defined to be cathodic. Simultaneously, at the zinc strip, zinc is dissolved into the solution,

$$
\mathrm{Zn} \rightarrow \mathrm{Zn}^{2+}+2 e^{-} .
$$

The reactant loses electrons, an oxidation takes place, and the strip is defined to be anodic.

In electrochemical cells, oxidation and reduction always occur simultaneously. As a result of the chemical reactions, the metal strips change shape. In the voltaic cell

Received December 1, 1995.

1991 Mathematics Subject Classification. Primary 35J20, 35J65, 73M99. 


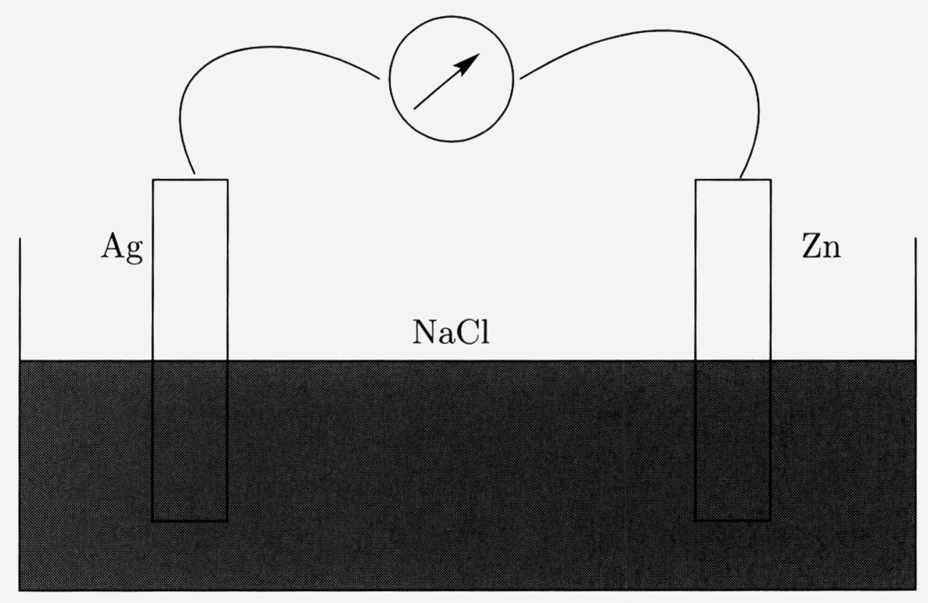

FIG. 1. Volta's cell

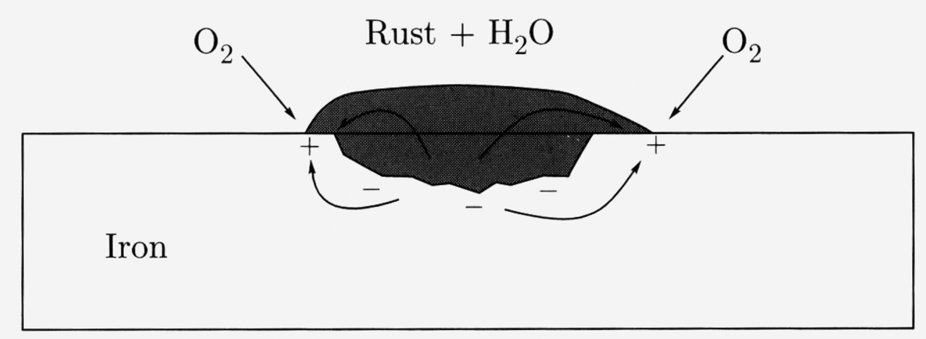

FIG. 2. A cell formed by rust on iron

illustrated above, the driving force for the oxidation-reduction reactions is the chemical potential difference between silver and zinc, and the chemical reactions produce a current. One may conversely use current generated by an outside power source to cause chemical reactions (involving electron transfer) at the metal surfaces. This alternate process is called electrolysis. The quantitative relationship between the electric current and the mass of substances consumed and produced by the chemical reactions has been studied intensively going back to the early work of Faraday.

Today, the use of electrochemical reactions plays a significant role in industry. Aside from electrochemical energy conversion, examples can be found in the production of chlorine, aluminum, and other chemicals, and in electroplating and electromachining. On the other hand, electrochemical phenomena also cause great damage, primarily in the form of corrosion. Corrosion is defined as the deterioration of a metal by chemical or electrochemical reaction with its environment. Most corrosion processes are electrochemical. In aqueous media, the action is similar to that taking place in the battery cell shown in Fig. 1 , where the zinc electrode is corroded, i.e., metallic zinc is converted into hydrated zinc ions $\mathrm{Zn}^{2+}$. Figure 2 illustrates a local cell on a piece of iron when it becomes rusted [16]. It is predominantly from the point of view of corrosion detection and corrosion control that we are interested in the problems associated with electrochemistry. To justify the 
commercial interest in these subjects it may be relevant to mention that a study conducted by the National Institute of Standards and Technology, under the request of the U.S. Congress, showed that the effect of corrosion cost the U.S. over 200 billion dollars in 1989 , which was approximately $4.2 \%$ of the U.S. gross national product of that year $[2]$.

The idealized electrochemical system we consider consists of a domain $\Omega$, containing an ionic solution (electrolyte). A part of the boundary of $\Omega$ is electrochemically active, the rest is electrochemically inactive (but may transmit an imposed current). For simplicity we restrict our attention to two-dimensional domains. We emphasize that the entire boundary is in contact with the same bulk solution, i.e., there are no membranes involved. One may imagine that outer circuits carry flows of electrons to and from the electrochemically active boundary parts, but exactly how this is done technically is of no importance for the analysis that we present here. Our main focus is to study the mathematical characteristics of a boundary value problem which incorporates a quite realistic model for the currents on the electrochemically active boundary parts. Since Faraday's law states that the rate of change of the boundary at any point is proportional to the normal current flux across the boundary at the point [5], a realistic modeling of the boundary fluxes thus indirectly provides a good understanding of the shape changes (corrosion) of the boundary.

We shall work entirely within the so-called potential model; we refer to [5], [12], [17] and the references therein, for a detailed derivation and justification of this model. Briefly stated the transport processes in the electrolytic solution are modeled by a dilute solution theory, in which the concentrations of the various species of the electrolytic solution are considered spatially constant away from the boundary. In this fashion one obtains a single elliptic equation for the electric potential, $\phi$,

$$
\nabla \cdot(-\kappa \nabla \phi)=0 \text { in } \Omega
$$

where $\kappa$, the conductivity of the solution, is given by

$$
\kappa=F^{2} \sum_{i} z_{i}^{2} u_{i} c_{i}
$$

In (2), $z_{i}$ is the charge number for the species $i, u_{i}$ is its mobility, and $c_{i}$ its concentration. Since the concentrations $c_{i}$ are assumed to be constant, so is the conductivity $\kappa$. Equation (1) therefore simply expresses that $\phi$ is harmonic in $\Omega$.

Let us now take a closer look at the interface between the electrochemically active boundary part and the electrolyte, where the oxidation-reduction reactions occur. As an aide to understanding the physical situation we consider for a moment a single strip of metal (anodic, say) placed in an electrolytic solution. After some period of time a state of equilibrium has been reached, at which point there is a discontinuity in the electrical potential across the metal-electrolyte interface (free electrons have gathered on the metal strip, metal ions have been released into the solution). The exact value of this potential jump depends on the specific metal and the specific electrolyte. Note that no net current is flowing between the metal and the electrolyte as a result of this potential jump.

Since the difference $\left(\phi_{\text {metal }}-\phi_{\text {solution }}\right)$ is not directly amenable to measurements, one introduces a reference device, such as a normal hydrogen electrode (NHE), to make a 


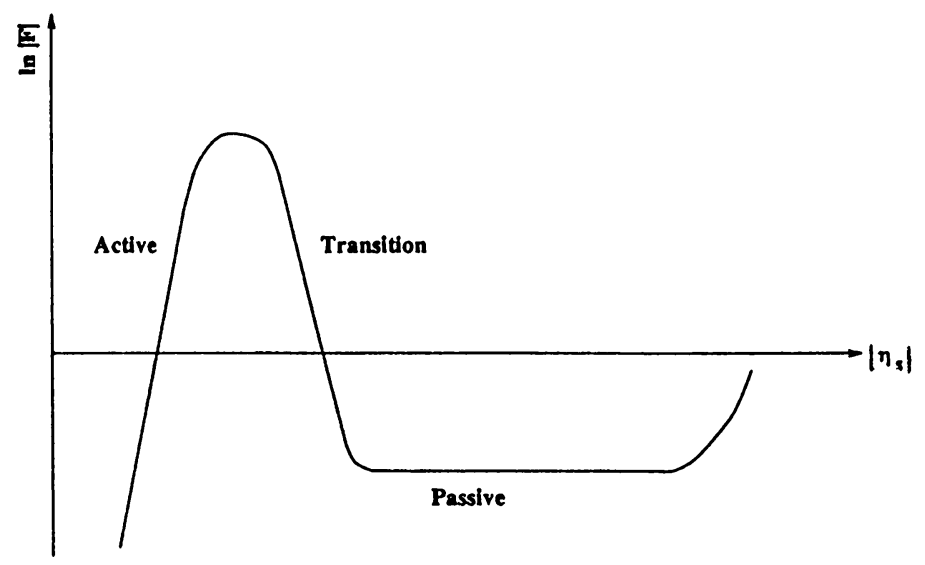

FIG. 3. Polarization curve

relative measurement. The relative potential of the metal strip with respect to an NHE placed in the solution is called its Nernst potential, $E_{N}$. If another strip of metal (of a different Nernst potential) is also placed in the solution, and if the two metal strips are connected by an outer circuit, then a current will flow through this circuit. At the metal-electrolyte interfaces, the potential differences will now be out of equilibrium. If one measures the potential of any one of the metal strips with respect to an NHE placed in the solution adjacent to the strip, then one obtains the galvanic potential of that strip, $E_{g}$. The difference $\eta_{s}=E_{g}-E_{N}$ is called the surface overpotential. It is the overpotential that is the driving force for the oxidation (or reduction) reaction at the metal-electrolyte interface. At each metal strip $\eta_{s}$ is functionally related to the current density

$$
\mathbf{J} \cdot \mathbf{n}=\mathcal{F}\left(\eta_{s}\right),
$$

where $\mathbf{J}$ is the current density and $\mathbf{n}$ is the unit outward normal (relative to the electrolyte, say). The exact form of $\mathcal{F}$ depends on the composition of the electrolyte near the interface and on the composition of the metal strip. For simplicity we suppose that there is only one reacting species in the electrolyte near each interface. In that case the composition of the electrolyte is locally characterized by the concentration, $c$, of this species.

In general, an analytical expression for $\mathcal{F}$ is not available, and approximations are usually obtained by interpolation of experimental data. The plot of $|\mathcal{F}|$ verses $\left|\eta_{s}\right|$ is called the polarization curve. Figure 3 illustrates a typical polarization curve (cf. [12]), where the vertical axis is $\ln |\mathcal{F}|$ and the horizontal axis is $\left|\eta_{s}\right|$. Quite often when $\eta_{s}$ is small, i.e., in the active region, the function $\mathcal{F}$ in (3) is well modeled by an exponential. At an anodic surface, $\eta_{s}$ is positive and $\mathcal{F}$ is negative; so

$$
\mathbf{J} \cdot \mathbf{n}=-J_{0} e^{\frac{\alpha z F}{R T} \eta_{s}} .
$$

At a cathodic surface $\eta_{s}$ is negative and $\mathcal{F}$ is positive; so

$$
\mathbf{J} \cdot \mathbf{n}=J_{0} e^{-\frac{(1-\alpha) z F}{R T} \eta_{s}} .
$$


Here $J_{0}>0$ is the exchange current density, which depends on the concentration $c$ (near the interface), on the composition of the metal surface, and on the temperature. $0<\alpha<1$ is a kinetic parameter, called the transfer coefficient, and $z$ (a positive integer) is the charge number of the dissolved or deposited species. $F$ is Faraday's constant, $R$ is the universal gas constant, and $T$ is absolute temperature. Combining the anodic and cathodic behaviour in one single boundary condition we obtain the so-called ButlerVolmer formula

$$
-\mathbf{J} \cdot \mathbf{n}=J_{0}\left(e^{\frac{\alpha z F}{R T} \eta_{s}}-e^{-\frac{(1-\alpha) z F}{R T} \eta_{s}}\right) .
$$

When $\eta_{s}$ is small and positive, the first term is leading and the formula closely approximates the anodic boundary condition discussed earlier. When $\eta_{s}$ is small and negative, the second term is leading and the formula closely approximates the cathodic boundary condition discussed earlier.

When $\eta_{s}$ is in the transition region, the reactions on the surface are complicated and unstable (see for instance [14] and the references therein). It may nonetheless in this region be reasonable to approximate the function, $\mathcal{F}$, by an expression very similar to that in (4), namely

$$
-\mathbf{J} \cdot \mathbf{n}=-J_{1}\left(e^{\frac{\tilde{\alpha} z F}{R T}\left(\eta_{s}-M\left(\eta_{s}\right)\right)}-e^{-\frac{(1-\tilde{\alpha})}{R T}\left(\eta_{s}-M\left(\eta_{s}\right)\right)}\right),
$$

with $J_{1}>0,0<\tilde{\alpha}<1$, and $M(t):=\operatorname{sign}(t) K$, for some positive constant $K$. We shall see that the boundary value problem corresponding to (5) is more difficult to analyze than that corresponding to (4); in particular, there may also exist multiple solutions.

Frequently when deriving realistic boundary conditions for the normal current one also includes an overpotential due to a diffusion layer. Such a diffusion layer is a region close to the boundary where the concentration, $c$, changes rapidly, and therefore the electric potential $\phi$ is not harmonic. Provided the thickness of this diffusion layer is small it is very reasonable to include the effects of this layer into the boundary condition for the normal current instead of changing the "interior" equation for $\phi$. The corresponding concentration overpotential is positive at the anodic part of the boundary, negative at the cathodic part. The concentration overpotential is simply added to the surface overpotential to form the total overpotential; we refer to ([5]) for a model of its relation to the normal current. In this paper, we have decided not to include the modeling of such a diffusion layer. It may be relevant here to note that under certain circumstances there are very simple experimental techniques to dramatically reduce the effect of the diffusion layer (cf. [9] and [10]).

As before let $\phi_{\text {metal }}$ denote the potential inside the metal that makes up the boundary and let $\phi$ denote the potential in the electrolyte. According to the previous discussion we then have

$$
\phi_{\text {metal }}(x)-\phi(x)=\eta_{s}(x)+E_{N}(x) \quad \text { on } \partial \Omega,
$$

or equivalently,

$$
\eta_{s}(x)=\phi_{\text {metal }}(x)-E_{N}(x)-\phi(x) \quad \text { on } \partial \Omega .
$$

In this context $h(x)=\phi_{\text {metal }}(x)-E_{N}(x), x \in \partial \Omega$, is an a priori given driving force for the internal voltage potential $\phi(x)$. We assume that $h(x)$ is a genuine driving force, that 
is, we assume that $h(x)$ is not constant on $\partial \Omega$. For $\eta_{s}$ in the active region, the nonlinear boundary value problem for the electrolytic voltage potential therefore becomes

$$
\begin{aligned}
-\Delta \phi & =0 \quad \text { in } \Omega, \\
\frac{\partial \phi}{\partial n} & =J_{0}\left(e^{\frac{\alpha z F}{R T}(h-\phi)}-e^{-\frac{(1-\alpha) z F}{R T}(h-\phi)}\right) \quad \text { on } \partial \Omega .
\end{aligned}
$$

Let $w$ denote the solution to

$$
\begin{aligned}
-\Delta w & =0 \quad \text { in } \Omega, \\
w & =h(x) \quad \text { on } \partial \Omega,
\end{aligned}
$$

and define $g=\left.\frac{z F}{R T} \frac{\partial w}{\partial n}\right|_{\partial \Omega}$. Since we have assumed that $h$ is not constant it follows that $w$ is not constant, and therefore that $g$ is not identically zero. At the same time we have that $\int_{\partial \Omega} g d l=\frac{z F}{R T} \int_{\Omega} \Delta w d x=0$. Let $v$ denote the function $v=\frac{z F}{R T}(w-\phi)$. This function satisfies the boundary value problem

$$
\begin{aligned}
-\Delta v & =0 \quad \text { in } \Omega, \\
\frac{\partial v}{\partial n} & =-J_{0} \frac{z F}{R T}\left(e^{\alpha v}-e^{-(1-\alpha) v}\right)+g \quad \text { on } \partial \Omega .
\end{aligned}
$$

If we consider $\eta_{s}$ entirely in the transition region and if we as a simplification think of $M\left(\eta_{s}\right)$ as a known function of $x, m(x)=M\left(\eta_{s}\right)$, then a calculation similar to that above, but with $h(x)=\phi_{\text {metal }}(x)-E_{N}(x)$ replaced by $h(x)=\phi_{\text {metal }}(x)-E_{N}(x)-m(x)$, gives that $v=\frac{z F}{R T}(w-\phi)$ satisfies the boundary value problem

$$
\begin{aligned}
-\Delta v & =0 \quad \text { in } \Omega, \\
\frac{\partial v}{\partial n} & =J_{1} \frac{z F}{R T}\left(e^{\alpha v}-e^{(1-\alpha) v}\right)+g \quad \text { on } \partial \Omega .
\end{aligned}
$$

Here we have used the notation $\alpha$ in place of $\tilde{\alpha}$. Instead of implicitly letting the electrochemically inactive part of the boundary correspond to that part where $v$ is zero or extremely close to zero we find it more convenient to explicitly prescribe the Neumann condition $\frac{\partial v}{\partial n}=g$ on some part of the boundary. Combining the above two boundary value problems into one, and taking into account the change in boundary condition just mentioned, we end up with the exact nonlinear boundary value problem, which we shall consider in the rest of this paper, namely

$$
\begin{aligned}
-\Delta u & =0 \quad \text { in } \Omega, \\
\frac{\partial u}{\partial n} & =\lambda\left(e^{\alpha u}-e^{-(1-\alpha) u}\right)+g \quad \text { on } \Gamma_{1}, \\
\frac{\partial u}{\partial n} & =g \quad \text { on } \Gamma_{2} .
\end{aligned}
$$

Here $\Omega \subset \mathbb{R}^{2}$ is a bounded, smooth domain and we suppose that $\Gamma_{1}$ and $\Gamma_{2}$ are two mutually disjoint, nonempty, connected, open subsets of $\partial \Omega$, with $\partial \Omega=\bar{\Gamma}_{1} \cup \bar{\Gamma}_{2}$. The parameter $\lambda$ is given by $\lambda=-J_{0} \frac{z F}{R T}<0$ or $\lambda=J_{1} \frac{z F}{R T}>0$, corresponding to the active region or the transition region, respectively. The positive constant $\alpha$ lies strictly between 0 and 1. The source term $g$ satisfies $\int_{\partial \Omega} g d l=0$. We introduce the functions

$$
F(x)=\frac{1}{\alpha} e^{\alpha x}+\frac{1}{1-\alpha} e^{-(1-\alpha) x}, \quad x \in \mathbb{R}
$$


(not to be confused with Faraday's constant) and

$$
f(x)=F^{\prime}(x)=e^{\alpha x}-e^{-(1-\alpha) x}, \quad x \in \mathbb{R} .
$$

Using $f$, we can write the boundary condition (7) as

$$
\frac{\partial u}{\partial n}=\lambda f(u)+g \quad \text { on } \Gamma_{1} .
$$

We shall consider the boundary value problem (6)-(8) in its natural weak formulation: find $u \in H^{1}(\Omega)$ such that

$$
\int_{\Omega} \nabla u \nabla v d x=\lambda \int_{\Gamma_{1}} f(u) v d l+\int_{\partial \Omega} g v d l
$$

for any $v \in H^{1}(\Omega)$. We always suppose that $g$ is in $L^{2}(\partial \Omega)$.

As we shall see in the beginning of the next section, the function $f(u)$ is in $L^{2}\left(\Gamma_{1}\right)$ if $u$ is in $H^{1}(\Omega)$. Standard elliptic regularity theory therefore implies that any weak solution, $u$, is $C^{\infty}$ inside $\Omega$ and globally is in $H^{3 / 2}(\Omega)$. The global regularity of $u$ gives that $\left.u\right|_{\Gamma_{1}}$ lies in $H^{1}\left(\Gamma_{1}\right)$ and since $H^{1}\left(\Gamma_{1}\right) \subset L^{\infty}\left(\Gamma_{1}\right)$ (remember, the boundary is one dimensional) it follows by differentiation that $f(u)$ lies in $H^{1}\left(\Gamma_{1}\right)$. If we now additionally required that $g$ be in $H^{1 / 2}(\partial \Omega)$, then elliptic regularity theory would imply that given any $y$ in the interior of $\Gamma_{1}$ or $\Gamma_{2}$ there exists a neighborhood of $y, \omega_{y}$, such that $u$ is in $H^{2}\left(\Omega \cap \omega_{y}\right)$. In addition to being a classical solution to $\Delta u=0$ inside $\Omega$ the weak solution $u$ would therefore satisfy the boundary conditions (7)-(8) in the sense of traces on any of the boundary parts $\partial \Omega \cap \omega_{y}$. If $g$ were to be smooth then $u$ would be smooth up to the boundary at any point interior to $\Gamma_{1}$ (or $\Gamma_{2}$ ), and the boundary conditions would be satisfied in a classical sense. There will in general be singularities of $u$ at the points that separate $\Gamma_{1}$ and $\Gamma_{2}$, even if $g$ is smooth.

The main theoretical results in this paper are proven in Secs. 1 and 2, and they may be summarized in the following.

TheOREM. Given $g \in L^{2}(\partial \Omega)$ there exists $\lambda_{0}>0$ such that the boundary value problem (11) has a solution for any $-\infty<\lambda<\lambda_{0}$. For $\lambda \leq 0$ this solution is unique (for $\lambda=0$, it is unique up to a constant). This unique solution may also be characterized as an energy minimizer. For $\lambda>0$ the problem (11) is likely to have multiple solutions; however, it is possible to construct a specific branch of solutions consisting of energy minimizers. The energy expressions differ for $\lambda<0$ and $\lambda>0$.

The Taylor expansion for the function $f$ at $x=0$ is given by

$$
f(x)=x+\frac{2 \alpha-1}{2} x^{2}+\cdots
$$

The boundary value problem (6)-(8) may thus be linearized near $u=0$ (and $g=0$ ) to give

$$
\begin{aligned}
-\Delta u & =0 \quad \text { in } \Omega, \\
\frac{\partial u}{\partial n} & =\lambda u+g \quad \text { on } \Gamma_{1} \\
\frac{\partial u}{\partial n} & =g \quad \text { on } \Gamma_{2} .
\end{aligned}
$$


The corresponding weak formulation becomes

$$
\int_{\Omega} \nabla u \nabla v d x=\lambda \int_{\Gamma_{1}} u v d l+\int_{\partial \Omega} g v d l .
$$

This linear problem has a unique solution when $\lambda$ lies outside a countable set of values $0<\lambda_{1}<\lambda_{2}<\cdots$, with $\left\{1 / \lambda_{n}\right\}_{n=1}^{\infty}$ representing the eigenvalues of an associated compact operator (for $\lambda=0$ the solution is unique up to a constant). In Sec. 3 of this paper we characterize the set $\left\{\lambda_{n}\right\}_{n=1}^{\infty}$ and we examine the structure of the solutions to (12) between these values. In particular, we characterize these intermediate solutions as solutions to appropriate minimization problems. In Sec. 4 we compare some numerical results obtained by the solution of the linear boundary value problem (12) with results obtained by the solution of the nonlinear boundary value problem (11). These numerical results also clearly indicate that the nonlinear boundary value problem has solutions for $\lambda$ moderately large and positive. It should be interesting to see if among these solutions it is still possible to select curves consisting of energy minimizers - but we have not succeeded in doing so. Finally, a short appendix is devoted to a survey of some fundamental facts concerning Orlicz spaces, which are needed in order to establish the existence of solutions for the nonlinear boundary value problem (11).

1. Existence and uniqueness for $\lambda \leq 0$. In this section we shall establish the existence and uniqueness of a solution to the boundary value problem (11) for values of $\lambda \leq 0$. For $\lambda=0$ the existence of a solution to the boundary value problem (11) is well known. It is equally well known that this solution is unique modulo a constant. It thus remains to consider $\lambda<0$. Let $I: H^{1}(\Omega) \rightarrow \mathbb{R}$ be the functional defined by

$$
I(u)=\frac{1}{2} \int_{\Omega}|\nabla u|^{2} d x-\lambda \int_{\Gamma_{1}} F(u) d l-\int_{\partial \Omega} g u d l .
$$

We shall now prove that $I$ is well defined and that it has a minimizer in $H^{1}(\Omega)$. This minimizer is easily seen to satisfy the Euler-Lagrange equation

$$
\int_{\Omega} \nabla u \nabla v d x=\lambda \int_{\Gamma_{1}} f(u) v d l+\int_{\partial \Omega} g v d l \quad \forall v \in H^{1}(\Omega),
$$

which is exactly the weak formulation (11). It is very easy to see that this solution is unique.

From the appendix at the end of this paper it follows that

$$
\|u\|_{L_{\Phi}\left(\Gamma_{1}\right)} \leq C\|u\|_{H^{1 / 2}\left(\Gamma_{1}\right)}
$$

where $\|\cdot\|_{L_{\Phi}}$ is the Luxemburg norm corresponding to the function

$$
\Phi(t)=e^{t^{2}}-1 \text {. }
$$

The definition of $\|\cdot\|_{L_{\Phi}\left(\Gamma_{1}\right)}$ implies that there is a positive real number $k$,

$$
\|u\|_{L_{\Phi}\left(\Gamma_{1}\right)} \leq k \leq\|u\|_{L_{\Phi}\left(\Gamma_{1}\right)}+1
$$

such that

$$
\int_{\Gamma_{1}} \Phi\left(\frac{u}{k}\right) d l \leq 1
$$


Insertion of the formula for $\Phi$ into this last inequality leads to

$$
\int_{\Gamma_{1}} e^{u^{2} / k^{2}} d l \leq \int_{\Gamma_{1}} 1 d l+1=\left|\Gamma_{1}\right|+1
$$

Combining (15) with the inequality

$$
\beta|u| \leq \frac{\beta^{2} k^{2}}{4}+\frac{u^{2}}{k^{2}}
$$

we find

$$
\int_{\Gamma_{1}} e^{\beta|u|} d l \leq e^{\beta^{2} k^{2} / 4}\left(\left|\Gamma_{1}\right|+1\right) \quad \text { for any } \beta \in \mathbb{R} .
$$

Due to the bounds (13) and (14) this immediately yields

$$
\int_{\Gamma_{1}} e^{\beta|u|} d l \leq e^{C \beta^{2}\left(\|u\|_{H^{1 / 2}\left(\Gamma_{1}\right)}^{2}+1\right)}\left(\left|\Gamma_{1}\right|+1\right) .
$$

For any $u \in H^{1}(\Omega)$,

$$
\|u\|_{H^{1 / 2}\left(\Gamma_{1}\right)} \leq\|u\|_{H^{1 / 2}(\partial \Omega)} \leq C\|u\|_{H^{1}(\Omega)}
$$

and hence

$$
\int_{\Gamma_{1}} e^{\beta|u|} d l \leq e^{C \beta^{2}\left(\|u\|_{H^{1}(\Omega)}^{2}+1\right)}\left(\left|\Gamma_{1}\right|+1\right) \quad \text { for any } \beta \in \mathbb{R},
$$

from which it follows that the functional $\int_{\Gamma_{1}} F(u) d l$, and thus the entire functional $I(u)$, is well defined on $H^{1}(\Omega)$. It also follows directly from (16) that $e^{\beta|u|}$ is in $L^{p}\left(\Gamma_{1}\right)$ for any $\beta \in \mathbb{R}$ and any $1 \leq p<\infty$. The inequality (16) is by no means new; it is for instance very similar to one found in [7], where one also finds an existence and uniqueness proof corresponding to $\lambda<0$. That proof is directly based on the convexity properties of the functional $I(\cdot)$, for $\lambda<0$. The existence and uniqueness proof we give here uses the convexity properties more indirectly since it proceeds by the "direct method" of the calculus of variations. We provide this simple argument, since a part of it is also used in our existence proof for the mathematically more interesting case of positive $\lambda$. We proceed to show that

$$
\inf _{u \in H^{1}(\Omega)} I(u)>-\infty
$$

and to show that any minimizing sequence has a subsequence that converges weakly in $H^{1}(\Omega)$. For any $d \in \mathbb{R}$ we find

$$
\left|\int_{\partial \Omega} g u d l\right|=\left|\int_{\partial \Omega} g(u+d) d l\right| \leq\|g\|_{L^{2}(\partial \Omega)}\|u+d\|_{L^{2}(\partial \Omega)} .
$$

At the same time,

$$
\min _{d \in \mathbb{R}}\|u+d\|_{L^{2}(\partial \Omega)} \leq C \min _{d \in \mathbb{R}}\|u+d\|_{H^{1}(\Omega)} \leq C\|\nabla u\|_{L^{2}(\Omega)} .
$$

The two previous estimates in combination yield

$$
\left|\int_{\partial \Omega} g u d l\right| \leq C\|g\|_{L^{2}(\partial \Omega)}\|\nabla u\|_{L^{2}(\Omega)} \leq \frac{C}{2 \varepsilon}\|g\|_{L^{2}(\partial \Omega)}^{2}+\frac{C \varepsilon}{2}\|\nabla u\|_{L^{2}(\Omega)}^{2},
$$


for any $\varepsilon>0$. From this estimate and the form of $I(u)$ it follows that there exist positive constants $c$ and $C$, independent of $u$, such that

$$
I(u) \geq c\|\nabla u\|_{L^{2}(\Omega)}^{2}-\lambda \int_{\Gamma_{1}} F(u) d l-C\|g\|_{L^{2}(\partial \Omega)}^{2} .
$$

Since the two first terms on the right-hand side are positive it is clear that $I(u) \geq$ $-C\|g\|_{L^{2}(\partial \Omega)}^{2}$, and thus inf $\operatorname{i\in H}_{u \in H^{1}(\Omega)} I(u)>-\infty$. Let $u_{n} \in H^{1}(\Omega)$ be a sequence such that

$$
I\left(u_{n}\right) \rightarrow \inf _{u \in H^{1}(\Omega)} I(u)
$$

Due to the estimate (19) there exists a constant $C$ such that

$$
\left\|\nabla u_{n}\right\|_{L^{2}(\Omega)} \leq C, \quad \forall n
$$

and consequently there exists a subsequence $u_{n_{k}}$ and a sequence of real number $c_{n_{k}}$ such that $u_{n_{k}}+c_{n_{k}}$ converges to some $\tilde{u}^{*}$ weakly in $H^{1}(\Omega)$. For simplicity we henceforth denote the index of this subsequence by $n$. We claim that the sequence $c_{n}$ is bounded. Since

$$
d|u| \leq F(u)
$$

for some $d>0$, and since $\lambda<0$ it follows from the bounds (19) and (20) that

$$
\int_{\Gamma_{1}}\left|u_{n}\right| d l \leq C, \quad \forall n
$$

The weak convergence of the sequence $u_{n}+c_{n}$ implies that it is bounded in $H^{1}(\Omega)$, and therefore

$$
\left\|u_{n}+c_{n}\right\|_{L^{2}(\partial \Omega)} \leq C, \quad \forall n .
$$

In particular, this implies that

$$
\int_{\Gamma_{1}}\left|u_{n}+c_{n}\right| d l \leq C, \quad \forall n
$$

Using (21) we now conclude

$$
\left|\Gamma_{1}\right|\left|c_{n}\right| \leq \int_{\Gamma_{1}}\left|u_{n}\right| d l+C \quad \forall n
$$

This boundedness of $c_{n}$ implies the existence of a convergent subsequence, which we for simplicity also index by $n$. The weak convergence of $u_{n}+c_{n}$ combined with the fact that the constants $c_{n}$ converge to $c^{*}$ yield that

$$
u_{n} \rightarrow u^{\star}=\tilde{u}^{*}-c^{*} \quad \text { in } H^{1}(\Omega) .
$$

We now proceed to show that $u^{\star}$ is a minimizer of $I(\cdot)$. The convexity of the functional

$$
\int_{\Omega}|\nabla u|^{2} d x
$$

and the fact that the $u_{n}$ converge weakly to $u^{\star}$ in $H^{1}(\Omega)$ give

$$
\int_{\Omega}\left|\nabla u^{\star}\right|^{2} d x \leq \liminf _{n} \int_{\Omega}\left|\nabla u_{n}\right|^{2} d x .
$$


Due to the weak convergence of the $u_{n}$ in $H^{1}(\Omega)$ it follows that $u_{n} \rightarrow u^{\star}$ in $H^{1 / 2}(\partial \Omega)$, and so

$$
\int_{\partial \Omega} g u^{\star} d l=\lim _{n} \int_{\partial \Omega} g u_{n} d l .
$$

Finally, we also have that

$$
\int_{\Gamma_{1}} F\left(u^{\star}\right) d l=\lim _{n} \int_{\Gamma_{1}} F\left(u_{n}\right) d l .
$$

To prove this it clearly suffices to prove that

$$
\int_{\Gamma_{1}} e^{\beta u^{\star}} d l=\lim _{n} \int_{\Gamma_{1}} e^{\beta u_{n}} d l
$$

for any $\beta \in \mathbb{R}$. Using the mean value theorem we find, for each $n$ and a.e. $x$, a value $\xi_{n}(x)$ with

$$
\min \left(u_{n}(x), u^{\star}(x)\right) \leq \xi_{n}(x) \leq \max \left(u_{n}(x), u^{\star}(x)\right)
$$

such that

$$
e^{\beta u_{n}}-e^{\beta u^{*}}=\beta e^{\beta \xi_{n}}\left(u_{n}-u^{\star}\right)
$$

The value $\xi_{n}(x)$ satisfies

$$
\left|\xi_{n}(x)\right| \leq\left|u_{n}(x)\right|+\left|u^{\star}(x)\right|
$$

and therefore it follows that

$$
\left|e^{\beta u_{n}}-e^{\beta u^{\star}}\right| \leq|\beta| e^{|\beta|\left(\left|u_{n}\right|+\left|u^{\star}\right|\right)}\left|u_{n}-u^{\star}\right| .
$$

Repeated use of the Schwartz inequality now yields

$$
\int_{\Gamma_{1}}\left|e^{\beta u_{n}}-e^{\beta u^{\star}}\right| d l \leq|\beta|\left\|e^{4\left|\beta u_{n}\right|}\right\|_{L^{1}\left(\Gamma_{1}\right)}^{1 / 4} \cdot\left\|e^{4\left|\beta u^{\star}\right|}\right\|_{L^{1}\left(\Gamma_{1}\right)}^{1 / 4} \cdot\left\|u_{n}-u^{\star}\right\|_{L^{2}\left(\Gamma_{1}\right)} .
$$

Since $u_{n}$ (and $u^{\star}$ ) are bounded in $H^{1}(\Omega)$, (16) shows that the first two norms on the right-hand side are bounded. The statement (26) follows from the fact that $u_{n}$ converges strongly to $u^{\star}$ in $L^{2}\left(\Gamma_{1}\right)$. A direct combination of the three statements (23), (24), and (25) now yields

$$
I\left(u^{\star}\right) \leq \lim _{n} I\left(u_{n}\right)=\inf _{u \in H^{1}(\Omega)} I(u),
$$

or in other words, $u^{\star}$ is a minimizer of $I(\cdot)$. This concludes the proof of existence of solutions to the boundary value problem (11) for $\lambda \leq 0$.

To conclude the study for $\lambda \leq 0$, we show that the solution to the problem (11) is unique if $\lambda<0$. Let $u^{*}$ and $u$ be two solutions to (11) for some $\lambda<0$. Then

$$
\int_{\Omega} \nabla\left(u^{*}-u\right) \nabla v d x=\lambda \int_{\Gamma_{1}}\left(f\left(u^{*}\right)-f(u)\right) v d l \quad \forall v \in H^{1}(\Omega),
$$

which by insertion of $v=u^{*}-u$ yields

$$
\int_{\Omega}\left|\nabla u^{*}-\nabla u\right|^{2} d x=\lambda \int_{\Gamma_{1}}\left(f\left(u^{*}\right)-f(u)\right)\left(u^{*}-u\right) d l .
$$


Since $f^{\prime}(x)=\alpha e^{\alpha x}+(1-\alpha) e^{-(1-\alpha) x}>0$ for any $x \in \mathbb{R}$, we have

$$
\left(f\left(u^{*}\right)-f(u)\right)\left(u^{*}-u\right) \geq 0 .
$$

It follows form (27), (28), and the fact that $\lambda \leq 0$ that

$$
\int_{\Omega}\left|\nabla u^{*}-\nabla u\right|^{2} d x \leq 0
$$

Consequently, $u=u^{*}+d$ for some constant $d$. For $\lambda<0$ any solution to the boundary value problem (11) obeys the condition $\int_{\Gamma_{1}} f(u) d l=0$ (simply insert $v=1$ into (11)). Therefore,

$$
\int_{\Gamma_{1}} f\left(u^{*}\right) d l=0 \quad \text { and } \quad \int_{\Gamma_{1}} f\left(u^{*}+d\right) d l=0,
$$

which immediately leads to $e^{\alpha d}=e^{-(1-\alpha) d}$, i.e., $d=0$ and $u=u^{*}$.

2. Existence for small $\lambda \geq 0$. In this section we consider the mathematically most interesting case, $\lambda \geq 0$. We prove that the weakly formulated boundary value problem (11) possesses a solution for $\lambda$ sufficiently small. This existence proof is based on a different minimization than that used in the previous section. A change of variational principle is clearly required, since the exponential character of the term $\int_{\Gamma_{1}} F(u) d l$ makes the functional $I(\cdot)$ unbounded from below, for $\lambda>0$. We introduce the functional

$$
\widetilde{J}(u)=\frac{1}{2} \int_{\Omega}|\nabla u|^{2} d x-\lambda \ln \int_{\Gamma_{1}} F(u) d l-\int_{\partial \Omega} g u d l .
$$

This functional is similar to that introduced in [11] for a related problem; we also refer to the introduction of [6] for a brief survey. The functional $\widetilde{J}(\cdot)$ is still unbounded from below: it is easy to see that $\widetilde{J}\left(c_{n}\right) \rightarrow-\infty$ for any sequence of constants, $c_{n}$, that converge to $+\infty$. We remove this latter unboundedness by defining

$$
J(u)=\sup _{r \in \mathbb{R}} \widetilde{J}(u+r),
$$

and we now seek a solution to the boundary value problem (11) by minimizing $J(\cdot)$ in $H^{1}(\Omega)$. In terms of the functional $\widetilde{J}$ we thus solve the saddle-point problem

$$
\inf _{u \in H^{1}(\Omega)} \sup _{r \in \mathbb{R}} \widetilde{J}(u+r) .
$$

It is not very difficult to calculate an explicit expression for the functional $J(\cdot)$. Indeed for any $u \in H^{1}(\Omega)$ and any $r \in \mathbb{R}$

$$
\widetilde{J}(u+r)=\frac{1}{2} \int_{\Omega}|\nabla u|^{2} d x-\lambda \ln \int_{\Gamma_{1}} F(u+r) d l-\int_{\partial \Omega} g u d l,
$$

and since $\lambda \geq 0$ and $\ln$ is a monotonically increasing function, the problem of maximizing $\widetilde{J}(u+r)$ in $r$ thus reduces to the problem of finding the constant $r$ that minimizes

$$
\int_{\Gamma_{1}} F(u+r) d l
$$


The expression (30) is well defined, continuous and always positive; furthermore, its value approaches $+\infty$ as $r \rightarrow \pm \infty$; so it attains its minimum. By taking the derivative with respect to $r$, we find that the minimizer $r^{\star}(u)$ must satisfy

$$
\int_{\Gamma_{1}} f\left(u+r^{\star}\right) d l=0
$$

the solution of which is given by

$$
e^{r^{\star}(u)}=\frac{\int_{\Gamma_{1}} e^{-(1-\alpha) u} d l}{\int_{\Gamma_{1}} e^{\alpha u} d l} .
$$

It follows from the discussion in the previous section that the expression on the righthand side is well defined and positive for any $u \in H^{1}(\Omega)$. Substitution of (31) back into the expression of the right-hand side of (29), now leads to

$$
\begin{aligned}
J(u)= & \sup _{r \in \mathbb{R}} \widetilde{J}(u+r)=\widetilde{J}\left(u+r^{\star}(u)\right) \\
= & \frac{1}{2} \int_{\Omega}|\nabla u|^{2} d x-\lambda(1-\alpha) \ln \int_{\Gamma_{1}} e^{\alpha u} d l-\lambda \alpha \ln \int_{\Gamma_{1}} e^{-(1-\alpha) u} d l \\
& \quad-\int_{\partial \Omega} g u d l+\lambda \ln \alpha(1-\alpha) .
\end{aligned}
$$

We proceed to study the minimization of $J(\cdot)$ in $H^{1}(\Omega)$. Due to the definition of $J$ it follows immediately that $J(u)=J(u+r)$ for any $r \in \mathbb{R}$. In order to prove that $\inf _{u \in H^{1}(\Omega)} J(u)>-\infty$ we shall in particular use that

$$
J(u)=J(u-\bar{u}),
$$

where

$$
\bar{u}=\frac{1}{|\Omega|} \int_{\Omega} u d x .
$$

Using the estimate (16) we find that

$$
\ln \int_{\Gamma_{1}} e^{\beta|u-\bar{u}|} d l \leq C \beta^{2}\left(\|u-\bar{u}\|_{H^{1}(\Omega)}^{2}+1\right)+\ln \left(\left|\Gamma_{1}\right|+1\right),
$$

for any $\beta \in \mathbb{R}$. It now follows from

$$
\|u-\bar{u}\|_{H^{1}(\Omega)} \leq C\|\nabla u\|_{L^{2}(\Omega)}
$$

that

$$
\ln \int_{\Gamma_{1}} e^{\beta|u-\bar{u}|} d l \leq C \beta^{2}\left(\|\nabla u\|_{L^{2}(\Omega)}^{2}+1\right)+\ln \left(\left|\Gamma_{1}\right|+1\right) .
$$

Combining (34) (for $\beta=\alpha$ and $\beta=1-\alpha$ ) with the estimate (18) and rearranging terms, we find that there exist positive constants $\lambda_{0}, c$, and $C$ such that

$$
J(u) \geq c\|\nabla u\|_{L^{2}(\Omega)}^{2}-C\left(\|g\|_{L^{2}\left(\Gamma_{1}\right)}^{2}+1\right),
$$

for any $0 \leq \lambda \leq \lambda_{0}$. This proves that $J$ is bounded from below for any $0 \leq \lambda \leq \lambda_{0}$. To show the existence of a minimizer, let $u_{n} \in H^{1}(\Omega)$ be a sequence such that

$$
J\left(u_{n}\right) \rightarrow \inf _{u \in H^{1}(\Omega)} J(u) .
$$


We may without loss of generality suppose that $\bar{u}_{n}=0$. The estimate (35) yields that $\left\|\nabla u_{n}\right\|_{L^{2}(\Omega)}$ is bounded, and hence that $\left\|u_{n}\right\|_{H^{1}(\Omega)}$ is bounded. We may now extract a subsequence of $u_{n}$ (for simplicity also indexed by $n$ ) that converges weakly to some $u^{*} \in H^{1}(\Omega)$, i.e.,

$$
u_{n} \rightarrow u^{\star} \quad \text { in } H^{1}(\Omega)
$$

It follows, as in the previous section, that

$$
J\left(u^{\star}\right) \leq \lim _{n} J\left(u_{n}\right) .
$$

Therefore, $u^{\star}$ is a minimizer of $J(\cdot)$ in $H^{1}(\Omega)$. In contrast to the last section it requires a little analysis to see that $u^{\star}$ gives rise to solutions to (11) for an interval of small positive $\lambda$. As part of this analysis we shall prove that the minimizer $u^{\star}$ is unique for $\lambda$ sufficiently small.

We now prove that for any $0 \leq \lambda \leq \lambda_{0}$ there exists a $0 \leq \tilde{\lambda}$ such that the function $\tilde{u}=u^{\star}+r^{*}\left(u^{\star}\right)$ is a solution of the boundary value problem (11) corresponding to the parameter $\tilde{\lambda}$. Here $r^{\star}\left(u^{\star}\right)$ is defined by (31). Simple manipulations give that the minimizer $u^{\star}$ satisfies the variational equation

$$
\begin{aligned}
\int_{\Omega} \nabla u^{\star} \nabla v d x-\frac{\lambda \alpha(1-\alpha)}{\int_{\Gamma_{1}} e^{\alpha u^{\star}} d l} & \int_{\Gamma_{1}} e^{\alpha u^{\star}} v d l \\
& +\frac{\lambda \alpha(1-\alpha)}{\int_{\Gamma_{1}} e^{-(1-\alpha) u^{\star}} d l} \int_{\Gamma_{1}} e^{-(1-\alpha) u^{*}} v d l-\int_{\partial \Omega} g v d l=0
\end{aligned}
$$

for any $v \in H^{1}(\Omega)$. In terms of $\tilde{u}=u^{\star}+r^{*}\left(u^{\star}\right)$ and the parameter

$$
\tilde{\lambda}=\lambda \alpha(1-\alpha)\left(\int_{\Gamma_{1}} e^{\alpha u^{\star}} d l\right)^{\alpha-1}\left(\int_{\Gamma_{1}} e^{-(1-\alpha) u^{\star}} d l\right)^{-\alpha} \geq 0
$$

this may be written

$$
\int_{\Omega} \nabla \tilde{u} \nabla v d x=\tilde{\lambda} \int_{\Gamma_{1}} f(\tilde{u}) v d l+\int_{\partial \Omega} g v d l \quad \forall v \in H^{1}(\Omega) .
$$

Thus $\tilde{u}$ is a solution of the problem (11), corresponding to the parameter value $\tilde{\lambda}$. It still remains to verify that the values $\tilde{\lambda}[\lambda]$ cover an entire interval $\left[0, \tilde{\lambda}_{0}\right]$, with $\tilde{\lambda}_{0}>0$, when $\lambda$ runs through an interval $\left[0, \lambda_{0}\right]$ with $\lambda_{0}>0$ sufficiently small. Since $\tilde{\lambda}[0]=0$ and $0<\tilde{\lambda}[\lambda]$ for $0<\lambda$, this follows immediately if we verify that the function $\tilde{\lambda}[\cdot]$ is continuous on $\left[0, \lambda_{0}\right]$ for $\lambda_{0}>0$ sufficiently small. In order to do so we first demonstrate that the minimizer $u^{\star}$ is unique (modulo a constant) for $\lambda$ sufficiently small.

At this point it becomes natural to emphasize that the functional $J(\cdot)=J_{\lambda}(\cdot)$ and any minimizer, $v^{*}=v_{\lambda}^{*}$, depend on $\lambda$. We suppose that $v_{\lambda}^{*}$ is normalized by

$$
\int_{\Omega} v_{\lambda}^{*} d x=0
$$

From the explicit expression for $J_{\lambda}(\cdot)$ and the lower bound (35) we now obtain

$$
\begin{aligned}
\lambda\left(\ln \alpha(1-\alpha)-\ln \left|\Gamma_{1}\right|\right) & =J_{\lambda}(0) \geq J_{\lambda}\left(v_{\lambda}^{*}\right) \\
& \geq c\left\|\nabla v_{\lambda}^{*}\right\|_{L^{2}(\Omega)}^{2}-C\left(\|g\|_{L^{2}\left(\Gamma_{1}\right)}^{2}+1\right),
\end{aligned}
$$


for $0 \leq \lambda \leq \lambda_{0}$. The constants $c$ and $C$ are positive and independent of $0 \leq \lambda \leq \lambda_{0}$, for $\lambda_{0}>0$ sufficiently small. This estimate immediately shows that there exists a constant $C$, independent of $\lambda$, such that

$$
\left\|\nabla v_{\lambda}^{*}\right\|_{L^{2}(\Omega)} \leq C, \quad 0 \leq \lambda \leq \lambda_{0}
$$

Combining this with the estimate (16), we immediately get that there exists a constant $C$, independent of $\lambda$, such that

$$
\int_{\Gamma_{1}} e^{-\alpha v_{\lambda}^{*}} d l \leq C, \quad 0 \leq \lambda \leq \lambda_{0} .
$$

Schwartz's inequality yields

$$
\left|\Gamma_{1}\right|=\int_{\Gamma_{1}} 1 d l \leq\left(\int_{\Gamma_{1}} e^{\alpha v_{\lambda}^{*}} d l\right)^{1 / 2}\left(\int_{\Gamma_{1}} e^{-\alpha v_{\lambda}^{*}} d l\right)^{1 / 2}
$$

and upon insertion of the bound (42), we now find that there exists a positive constant, $c$, independent of $\lambda$, such that

$$
\int_{\Gamma_{1}} e^{\alpha v_{\lambda}^{*}} d l>c, \quad 0 \leq \lambda \leq \lambda_{0}
$$

Similarly, we can show that

$$
\int_{\Gamma_{1}} e^{-(1-\alpha) v_{\lambda}^{*}} d l>c, \quad 0 \leq \lambda \leq \lambda_{0}
$$

Based on the definition of $\tilde{\lambda},(38)$, we conclude that there exists a constant, $C$, so that $0 \leq \tilde{\lambda} \leq C$ for $0 \leq \lambda \leq \lambda_{0}$. Since the function $v_{\lambda}^{*}$ is a minimizer of $J_{\lambda}$, the function $\tilde{v}_{\lambda}=v_{\lambda}^{*}+r^{*}\left(v_{\lambda}^{*}\right)$ satisfies the variational equation (39) where $\tilde{\lambda}$ is given by the expression (38) with $u^{\star}$ replaced by $v_{\lambda}^{*}$. The functions $f\left(\tilde{v}_{\lambda}\right)$ and $g$ are in $L^{2}\left(\Gamma_{1}\right)$ and $L^{2}(\partial \Omega)$ respectively, and hence it follows from classical regularity estimates for linear elliptic boundary value problems that $\tilde{v}_{\lambda}$ is in $H^{3 / 2}(\Omega)$. Furthermore, since $\tilde{\lambda}$ is uniformly bounded, it follows that

$$
\left\|\tilde{v}_{\lambda}\right\|_{H^{3 / 2}(\Omega)} \leq C, \quad 0 \leq \lambda \leq \lambda_{0}
$$

with a constant $C$, which is independent of $\lambda$. Let now $u_{\lambda}^{\star}$ and $v_{\lambda}^{\star}$ denote two minimizers of $J_{\lambda}$, normalized by

$$
\int_{\Omega} u_{\lambda}^{\star} d x=\int_{\Omega} v_{\lambda}^{\star} d x=0 .
$$

Since $u_{\lambda}^{\star}$ and $v_{\lambda}^{\star}$ both satisfy the variational equation (37) we get

$$
\int_{\Omega} \nabla\left(u_{\lambda}^{\star}-v_{\lambda}^{\star}\right) \nabla v d x=\lambda \int_{\Gamma_{1}}\left(f^{*}\left(u_{\lambda}^{\star}\right)-f^{*}\left(v_{\lambda}^{\star}\right)\right) v d l \quad \forall v \in H^{1}(\Omega),
$$

where the function $f^{*}(u)(x)$ is given by the formula

$$
f^{\star}(u)(x)=\alpha(1-\alpha)\left(\frac{e^{\alpha u(x)}}{\int_{\Gamma_{1}} e^{\alpha u} d l}-\frac{e^{-(1-\alpha) u(x)}}{\int_{\Gamma_{1}} e^{-(1-\alpha) u} d l}\right) .
$$


Taking $v=u_{\lambda}^{\star}-v_{\lambda}^{\star}$ in (46) we obtain

$$
\int_{\Omega}\left|\nabla u_{\lambda}^{\star}-\nabla v_{\lambda}^{\star}\right|^{2} d x=\lambda \int_{\Gamma_{1}}\left(f^{\star}\left(u_{\lambda}^{\star}\right)-f^{\star}\left(v_{\lambda}^{\star}\right)\right)\left(u_{\lambda}^{\star}-v_{\lambda}^{\star}\right) d l .
$$

If we combine the identity

$$
f^{\star}(u)-f^{\star}(v)=\int_{0}^{1} \frac{d}{d t} f^{\star}(v+t(u-v)) d t
$$

with the explicit formula

$$
\begin{aligned}
& \frac{d}{d t} f^{\star}(v+t(u-v)) \\
& =\alpha^{2}(1-\alpha) \frac{e^{\alpha(v+t(u-v))}(u-v)}{\int_{\Gamma_{1}} e^{\alpha(v+t(u-v))} d l} \\
& \quad-\alpha^{2}(1-\alpha) \frac{e^{\alpha(v+t(u-v))} \int_{\Gamma_{1}} e^{\alpha(v+t(u-v))}(u-v) d l}{\left(\int_{\Gamma_{1}} e^{\alpha(v+t(u-v))} d l\right)^{2}} \\
& \quad+\alpha(1-\alpha)^{2} \frac{e^{-(1-\alpha)(v+t(u-v))}(u-v)}{\int_{\Gamma_{1}} e^{-(1-\alpha)(v+t(u-v))} d l} \\
& \quad-\alpha(1-\alpha)^{2} \frac{e^{-(1-\alpha)(v+t(u-v))} \int_{\Gamma_{1}} e^{-(1-\alpha)(v+t(u-v))}(u-v) d l}{\left(\int_{\Gamma_{1}} e^{-(1-\alpha)(v+t(u-v))} d l\right)^{2}}
\end{aligned}
$$

insert the result into (47), and change the order of integration, then we immediately get

$$
\begin{aligned}
\int_{\Omega} \mid \nabla u_{\lambda}^{\star} & -\left.\nabla v_{\lambda}^{\star}\right|^{2} d x \\
=\lambda & \int_{0}^{1}\left[\alpha^{2}(1-\alpha) \frac{\int_{\Gamma_{1}} e^{\alpha\left(v_{\lambda}^{\star}+t\left(u_{\lambda}^{\star}-v_{\lambda}^{\star}\right)\right)}\left(u_{\lambda}^{\star}-v_{\lambda}^{\star}\right)^{2} d l}{\int_{\Gamma_{1}} e^{\alpha\left(v_{\lambda}^{\star}+t\left(u_{\lambda}^{\star}-v_{\lambda}^{\star}\right)\right)} d l}\right. \\
& -\alpha^{2}(1-\alpha) \frac{\left(\int_{\Gamma_{1}} e^{\alpha\left(v_{\lambda}^{\star}+t\left(u_{\lambda}^{\star}-v_{\lambda}^{\star}\right)\right)}\left(u_{\lambda}^{\star}-v_{\lambda}^{\star}\right) d l\right)^{2}}{\left(\int_{\Gamma_{1}} e^{\alpha\left(v_{\lambda}^{\star}+t\left(u_{\lambda}^{\star}-v_{\lambda}^{\star}\right)\right)} d l\right)^{2}} \\
& +\alpha(1-\alpha)^{2} \frac{\int_{\Gamma_{1}} e^{-(1-\alpha)\left(v_{\lambda}^{\star}+t\left(u_{\lambda}^{\star}-v_{\lambda}^{\star}\right)\right)}\left(u_{\lambda}^{\star}-v_{\lambda}^{\star}\right)^{2} d l}{\int_{\Gamma_{1}} e^{-(1-\alpha)\left(v_{\lambda}^{*}+t\left(u_{\lambda}^{\star}-v_{\lambda}^{\star}\right)\right)} d l} \\
& \left.-\alpha(1-\alpha)^{2} \frac{\left(\int_{\Gamma_{1}} e^{-(1-\alpha)\left(v_{\lambda}^{*}+t\left(u_{\lambda}^{\star}-v_{\lambda}^{\star}\right)\right)}\left(u_{\lambda}^{\star}-v_{\lambda}^{\star}\right) d l\right)^{2}}{\left(\int_{\Gamma_{1}} e^{-(1-\alpha)\left(v_{\lambda}^{\star}+t\left(u_{\lambda}^{\star}-v_{\lambda}^{\star}\right)\right)} d l\right)^{2}}\right] d t .
\end{aligned}
$$

It follows from (45) that

$$
\left\|u_{\lambda}^{\star}\right\|_{L^{\infty}\left(\Gamma_{1}\right)} \leq C\left\|u_{\lambda}^{\star}\right\|_{H^{3 / 2}(\Omega)} \leq C^{\prime}, \quad 0 \leq \lambda \leq \lambda_{0} .
$$

The same estimate holds for $v_{\lambda}^{\star}$. Employing a technique similar to that which we used earlier to prove the lower bounds (43) and (44), we find that all the terms that appear in the denominators in (48) are bounded uniformly away from 0 for $0 \leq \lambda \leq \lambda_{0}$ and $0 \leq t \leq 1$. These lower bounds in combination with the estimate (49) justify the validity 
of the change of order of integration that led to (48); but more importantly, because of the form of the identity (48) they lead to the existence of a constant $C$, independent of $\lambda$, such that

$$
\int_{\Omega}\left|\nabla u_{\lambda}^{\star}-\nabla v_{\lambda}^{\star}\right|^{2} d x \leq \lambda C \int_{\Gamma_{1}}\left(u_{\lambda}^{\star}-v_{\lambda}^{\star}\right)^{2} d l
$$

for all $0 \leq \lambda \leq \lambda_{0}$. From the Poincaré estimate (33) and the fact that $\bar{u}_{\lambda}^{*}=\bar{v}_{\lambda}^{*}=0$ we now obtain

$$
\int_{\Omega}\left|\nabla u_{\lambda}^{\star}-\nabla v_{\lambda}^{\star}\right|^{2} d x \leq \lambda C \int_{\Omega}\left|\nabla u_{\lambda}^{\star}-\nabla v_{\lambda}^{\star}\right|^{2} d x
$$

with $C$ independent of $0 \leq \lambda \leq \lambda_{0}$. For $\lambda<1 / C$ this implies that $u_{\lambda}^{\star}=v_{\lambda}^{\star}$. Therefore there exists $0<\lambda_{0}$ such that $J_{\lambda}(\cdot)$ has a unique minimizer (modulo a constant) for $0 \leq \lambda \leq \lambda_{0}$. We note that we have only proved the uniqueness of the minimizer of $J_{\lambda}$ (which in turn leads to a particular solution to the boundary value problem (11) for parameter value $\tilde{\lambda}$ ). We have not proved uniqueness of solutions to the boundary value problem (11), and indeed numerical evidence strongly suggests that such uniqueness is not true.

We are now ready to prove that $\tilde{\lambda}$ depends continuously on $\lambda \in\left[0, \lambda_{0}\right]$. The key here is to show that for any $\lambda_{n}, \lambda \in\left[0, \lambda_{0}\right]$, the fact that $\lambda_{n} \rightarrow \lambda$ implies that

$$
u_{\lambda_{n}}^{\star} \rightarrow u_{\lambda}^{\star} \text { in } H^{1}(\Omega) \text {, }
$$

where $u_{\lambda}^{\star}$ denotes the unique minimizer of $J_{\lambda}$ with $\bar{u}_{\lambda}^{*}=0$ (and similarly for $\lambda_{n}$ ). The fact that $\tilde{\lambda}\left[\lambda_{n}\right] \rightarrow \tilde{\lambda}[\lambda]$ then follows directly from the formula (38). It is already known that

$$
\left\|\nabla u_{\lambda_{n}}^{\star}\right\|_{L^{2}(\Omega)} \leq C
$$

uniformly in $n$; it is also known that one has the bounds

$$
0<c<\int_{\Gamma_{1}} e^{\alpha u_{\lambda_{n}}^{*}} d l, \quad \int_{\Gamma_{1}} e^{-(1-\alpha) u_{\lambda_{n}}^{\star}} d l<C
$$

uniformly in $n$. As a consequence of (52) there exists a subsequence (also dentoed $u_{\lambda_{n}}^{\star}$ ) and some $u \in H^{1}(\Omega)$ such that

$$
u_{\lambda_{n}}^{\star} \rightarrow u \text { in } H^{1}(\Omega) .
$$

To verify the convergence (51) for the entire sequence, it suffices to show that $u=u_{\lambda}^{\star}$. Due to the (previously established) weak lower semicontinuity of the functional $J_{\lambda}(\cdot)$ and due to the bounds (53) it follows that

$$
J_{\lambda}(u) \leq \lim _{n} J_{\lambda}\left(u_{\lambda_{n}}^{\star}\right)=\lim _{n} J_{\lambda_{n}}\left(u_{\lambda_{n}}^{\star}\right) .
$$

It is also clear that

$$
J_{\lambda}\left(u_{\lambda}^{\star}\right)=\lim _{n} J_{\lambda_{n}}\left(u_{\lambda}^{\star}\right) .
$$

Since $u_{\lambda_{n}}^{\star}$ is the minimizer of $J_{\lambda_{n}}$, we have

$$
J_{\lambda_{n}}\left(u_{\lambda_{n}}^{\star}\right) \leq J_{\lambda_{n}}\left(u_{\lambda}^{\star}\right)
$$


If we now let $n \rightarrow \infty$ and combine (54), (55), and (56), then

$$
J_{\lambda}(u) \leq \lim _{n} J_{\lambda_{n}}\left(u_{\lambda_{n}}^{\star}\right) \leq \lim _{n} J_{\lambda_{n}}\left(u_{\lambda}^{\star}\right)=J_{\lambda}\left(u_{\lambda}^{\star}\right) .
$$

By uniqueness of the minimizer with integral zero, we thus obtain $u=u_{\lambda}^{\star}$. This concludes the proof of existence of solutions to the boundary value problem (11) for $0 \leq \lambda \leq \lambda_{0}$ with $\lambda_{0}>0$ sufficiently small.

REMARK. It might be relevant to note that there are other approaches one could use to establish the existence of solutions to the problem (11) for sufficiently small positive $\lambda$. One could for instance use an approach based on an Implicit Function Theorem such as that found in Chapter 1 of [4]. Such an approach would establish the existence of a curve of solutions, but it would not lead to a global characterization of this particular curve of solutions (as obtained from energy minimization).

3. Linear theory. In this section we study the behaviour of the solutions to the linear boundary value problem (12), as $\lambda$ varies. For simplicity we shall from now on always select the boundary flux $g \in L^{2}(\partial \Omega)$ such that $g=0$ on $\Gamma_{1}$ (and therefore $\left.\int_{\Gamma_{2}} g d l=0\right)$. We thus consider the solutions to

$$
\int_{\Omega} \nabla u_{\lambda} \nabla v d x=\lambda \int_{\Gamma_{1}} u_{\lambda} v d l+\int_{\Gamma_{2}} g v d l \quad \forall v \in H^{1}(\Omega) .
$$

In order to carry out our study it is convenient to define a linear operator, $T$, from the space

$$
\widetilde{L}^{2}\left(\Gamma_{1}\right)=L^{2}\left(\Gamma_{1}\right) \cap\left\{\int_{\Gamma_{1}} \eta d l=0\right\}
$$

to itself. Given any $\eta \in \widetilde{L}^{2}\left(\Gamma_{1}\right)$ let $S(\eta) \in H^{1}(\Omega)$ denote the solution to the weakly formulated Neumann problem

$$
\int_{\Omega} \nabla S(\eta) \nabla v d x=\int_{\Gamma_{1}} \eta v d l \quad \forall v \in H^{1}(\Omega),
$$

satisfying the integral condition

$$
\int_{\Gamma_{1}} S(\eta) d l=0
$$

Define

$$
T \eta=\left.S(\eta)\right|_{\Gamma_{1}}
$$

The operator $T$ is one-to-one, positive definite and selfadjoint. Since $\left.S(\eta)\right|_{\Gamma_{1}}$ is in $H^{1 / 2}\left(\Gamma_{1}\right), T: \widetilde{L}^{2}\left(\Gamma_{1}\right) \rightarrow \widetilde{L}^{2}\left(\Gamma_{1}\right)$ is also compact. We remark that, for $\lambda \neq 0$, any solution of (57) automatically satisfies

$$
\int_{\Gamma_{1}} u_{\lambda} d l=0
$$

For $\lambda=0$ the solution to (57), $u_{0}$, is only defined up to a constant; we may select this constant so that the above integral constraint holds. If we write $w_{\lambda}=u_{\lambda}-u_{0}$, then $w_{\lambda}$ 
is a solution to

$$
\int_{\Omega} \nabla w_{\lambda} \nabla v d x=\lambda \int_{\Gamma_{1}}\left(w_{\lambda}+u_{0}\right) v d l \quad \forall v \in H^{1}(\Omega),
$$

satisfying the integral constraint

$$
\int_{\Gamma_{1}} w_{\lambda} d l=0
$$

Using the operator $T$ this is equivalent to saying that $\left.w_{\lambda}\right|_{\Gamma_{1}}$ is a solution to

$$
\lambda T\left(w_{\lambda}\left|\Gamma_{1}+u_{0}\right|_{\Gamma_{1}}\right)=\left.w_{\lambda}\right|_{\Gamma_{1}} .
$$

For $\lambda=0$ there is a unique solution, $w_{0}=0$. For $\lambda \neq 0$ we rearrange terms to obtain

$$
\left.\left(T-\frac{1}{\lambda} I\right) w_{\lambda}\right|_{\Gamma_{1}}=-T\left(\left.u_{0}\right|_{\Gamma_{1}}\right) \text {. }
$$

This problem has a unique solution exactly when $1 / \lambda$ is not an eigenvalue of $T$. If $1 / \lambda$ is an eigenvalue of $T$, then the problem is solvable if and only if $T\left(\left.u_{0}\right|_{\Gamma_{1}}\right)$ is orthogonal to the eigenfunctions of $T$ associated with $1 / \lambda$ (in the usual $L^{2}\left(\Gamma_{1}\right)$ inner product) and the solution is only determined up to a linear combination of eigenfunctions. We note that since $T$ is selfadjoint, and since 0 is not an eigenvalue of $T, T\left(\left.u_{0}\right|_{\Gamma_{1}}\right)$ is orthogonal to a particular eigenfunction, $\phi$, if and only if $\left.u_{0}\right|_{\Gamma_{1}}$ is orthogonal to that same eigenfunction. In terms of $g$ it is not very difficult to see that $\left.u_{0}\right|_{\Gamma_{1}}$ is orthogonal to an eigenfunction $\phi$ if and only if $g$ is orthogonal to $\left.S(\phi)\right|_{\Gamma_{2}}$ (in the usual $L^{2}\left(\Gamma_{2}\right)$ inner product). In terms of the original boundary value problem for $u_{\lambda}$, this may be restated to say that (57) has a unique solution exactly when $1 / \lambda$ is not an eigenvalue of $T$. If $1 / \lambda$ is an eigenvalue then there is a solution if and only if the source term $g$ is orthogonal to all the functions $S(\phi)$ where $\phi$ is an eigenfunction associated with $1 / \lambda$ (or, equivalently, if $\left.u_{0}\right|_{\Gamma_{1}}$ is orthogonal to all such $\phi$ ). This in particular means that for any $g$ that is not identically zero on $\Gamma_{2}$ (but vanishes on $\Gamma_{1}$ ) there exists at least one (positive) value $\lambda$ for which (57) has no solution. If indeed (57) had a solution for all $\lambda>0$ then $\left.u_{0}\right|_{\Gamma_{1}}$ would be orthogonal to all eigenfunctions of $T$. Since the eigenfunctions of $T$ form an orthogonal basis for $L^{2}\left(\Gamma_{1}\right)$ it follows that $\left.u_{0}\right|_{\Gamma_{1}}=0$, and hence

$$
u_{0}=\frac{\partial u_{0}}{\partial n}=0 \quad \text { on } \Gamma_{1} .
$$

Since $\Delta u_{0}=0$ in $\Omega$, we conclude from the unique continuation property of the Laplacian that $u_{0}=0$ in all of $\Omega$. Due to (57) this implies that $g=0$, which clearly represents a contradiction. In the previous argument we have used the fact that standard elliptic regularity theory ensures that $u_{0}$ is smooth inside $\Omega$, and is also smooth up to the boundary near any interior point of $\Gamma_{1}$.

In order to better understand the structure of solutions to the linear boundary value problem (57) we provide a characterization in terms of appropriate minimization problems. Let $E_{i}$ denote the eigenspace corresponding to the $i$ th eigenvalue of $T$ (numbered in decreasing order). We initially suppose that $g$ is not orthogonal to either the space $\left.S\left(E_{1}\right)\right|_{\Gamma_{2}}$ or the space $\left.S\left(E_{2}\right)\right|_{\Gamma_{2}}\left(\right.$ in $\left.L^{2}\left(\Gamma_{2}\right)\right)$. Let $\langle\cdot, \cdot\rangle$ denote the inner product

$$
\langle u, v\rangle=\int_{\Gamma_{1}} u v d l
$$


and let $B_{0}$ denote the set

$$
B_{0}=H^{1}(\Omega) \cap\{\langle v, 1\rangle=0,\langle v, v\rangle=1\} .
$$

For each $0<\mu<\infty$, the functional $I_{\mu}$ is defined by

$$
I_{\mu}(v)=\frac{1}{2} \int_{\Omega}|\nabla v|^{2} d x-\frac{1}{\mu} \int_{\Gamma_{2}} g v d l .
$$

Consider now the minimization problem

$$
\min _{v \in B_{0}} I_{\mu}(v)
$$

This problem has a unique minimizer with minimal $\int_{\Omega}|\nabla \cdot|^{2} d x$, and it is easy to see that this minimizer, $\tilde{u}_{\mu}$, also solves

$$
\int_{\Omega} \nabla \tilde{u}_{\mu} \nabla v d x=\lambda(\mu) \int_{\Gamma_{1}} \tilde{u}_{\mu} v d l+\frac{1}{\mu} \int_{\Gamma_{2}} g v d l \quad \forall v \in H^{1}(\Omega),
$$

with

$$
\lambda(\mu)=\int_{\Omega}\left|\nabla \tilde{u}_{\mu}\right|^{2} d x-\frac{1}{\mu} \int_{\Gamma_{2}} g \tilde{u}_{\mu} d l .
$$

It is not difficult to see that $\lambda(\mu)$ depends continuously on $\mu$ and that

$$
-\infty<\lambda(\mu)<\lambda_{1} \quad \text { for } 0<\mu<\infty
$$

with

$$
\lambda(\mu) \rightarrow-\infty \text { as } \mu \rightarrow 0 \text { and } \lambda(\mu) \rightarrow \lambda_{1} \text { as } \mu \rightarrow \infty .
$$

Here $1 / \lambda_{1}$ is the largest eigenvalue of the operator $T$. If we define $u=\mu \tilde{u}_{\mu}$, then it is clear that $u$ is the solution to (57) with $\lambda=\lambda(\mu)$. The formula (58) therefore provides an alternate variational characterization of the solution to the linear boundary value problem (57) for $-\infty<\lambda<\lambda_{1}$. For $\lambda=\lambda_{1}$ there is, as discussed above, no solution to (57).

To characterize the solution of (57) for $\lambda_{1}<\lambda<\lambda_{2}$ (where $1 / \lambda_{2}$ is the second largest eigenvalue of $T$ ) it is possible to derive an analogous minimization problem. At this point we suppose that $g$ is not orthogonal to any of the spaces $\left.S\left(E_{1}\right)\right|_{\Gamma_{2}},\left.S\left(E_{2}\right)\right|_{\Gamma_{2}}$, or $\left.S\left(E_{3}\right)\right|_{\Gamma_{2}}$. Define the set

$$
B_{1}=H^{1}(\Omega) \cap\left\{\langle v, 1\rangle=0,\langle v, \phi\rangle=0 \forall \phi \in E_{1},\langle v, v\rangle=1\right\} .
$$

For any fixed $0<\mu<\infty$ consider now the minimization problem

$$
\min _{v \in B_{1}} I_{\mu}(v)
$$

where $I_{\mu}$ is as defined previously. The minimizer, $\tilde{u}_{\mu}$, with minimal $\int_{\Omega}|\nabla \cdot|^{2} d x$, satisfies the equation

$$
\int_{\Omega} \nabla \tilde{u}_{\mu} \cdot \nabla v d x=\lambda(\mu) \int_{\Gamma_{1}} \tilde{u}_{\mu} v d l+\frac{1}{\mu} \int_{\Gamma_{2}} g v d l
$$

for any $v \in H^{1}(\Omega)$ with $\langle v, \phi\rangle=0 \forall \phi \in E_{1}$. Here $\lambda(\mu)$ is given by

$$
\lambda(\mu)=\int_{\Omega}\left|\nabla \tilde{u}_{\mu}\right|^{2} d x-\frac{1}{\mu} \int_{\Gamma_{2}} g \tilde{u}_{\mu} d l .
$$


Suppose now for simplicity that the eigenvalue $1 / \lambda_{1}$ has multiplicity 1 , i.e., $E_{1}=$ $\operatorname{span}\left\{\phi_{1}\right\}$, where $\phi_{1}$ is a single function, say with $\left\langle\phi_{1}, \phi_{1}\right\rangle=1$. Let $v_{1} \in H^{1}(\Omega)$ denote the solution to

$$
\int_{\Omega} \nabla v_{1} \nabla v d x=\int_{\Gamma_{1}} \phi_{1} v d l \quad \forall v \in H^{1}(\Omega),
$$

in other words $v_{1}=S\left(\phi_{1}\right)$. Due to the definition of the operator $T$ we have $\left.v_{1}\right|_{\Gamma_{1}}=T \phi_{1}=$ $\left(1 / \lambda_{1}\right) \phi_{1}$; as a consequence $\left\langle v_{1}, 1\right\rangle=0$ and $\left\langle v_{1}, v_{1}\right\rangle=1 / \lambda_{1}^{2}$. Any vector $v \in H^{1}(\Omega)$ may now be decomposed as

$$
v=w+\lambda_{1}^{2}\left\langle v, v_{1}\right\rangle v_{1},
$$

where the function $w$ satisfies $\langle w, \phi\rangle=0 \forall \phi \in E_{1}$. To make the system governing the minimizer, $\tilde{u}_{\mu}$, more familiar we use $(60),(61)$, and (62) to compute

$$
\begin{aligned}
\int_{\Omega} \nabla \tilde{u}_{\mu} \nabla v d x & =\int_{\Omega} \nabla \tilde{u}_{\mu} \nabla w d x+\lambda_{1}^{2}\left\langle v, v_{1}\right\rangle \int_{\Omega} \nabla \tilde{u}_{\mu} \nabla v_{1} d x \\
& =\lambda(\mu) \int_{\Gamma_{1}} \tilde{u}_{\mu} w d l+\frac{1}{\mu} \int_{\Gamma_{2}} g w d l \\
& =\lambda(\mu) \int_{\Gamma_{1}} \tilde{u}_{\mu} v d l+\frac{1}{\mu} \int_{\Gamma_{2}} g v d l-\frac{\lambda_{1}^{2}\left\langle v, v_{1}\right\rangle}{\mu} \int_{\Gamma_{2}} g v_{1} d l \\
& =\lambda(\mu) \int_{\Gamma_{1}} \tilde{u}_{\mu} v d l+\frac{1}{\mu} \int_{\Gamma_{2}} g v d l-\frac{\lambda_{1}^{2} \int_{\Gamma_{2}} g S\left(\phi_{1}\right) d l}{\mu} \int_{\Gamma_{1}} v_{1} v d l .
\end{aligned}
$$

If we now define $u=\mu \tilde{u}_{\mu}-\gamma v_{1}$, then we obtain

$$
\begin{aligned}
\int_{\Omega} \nabla u \nabla v d x=\lambda(\mu) \int_{\Gamma_{1}} u v d l+\int_{\Gamma_{2}} g v d l & \\
& +\left[\gamma\left(\lambda(\mu)-\lambda_{1}\right)-\lambda_{1}^{2} \int_{\Gamma_{2}} g S\left(\phi_{1}\right) d l\right] \int_{\Gamma_{1}} v_{1} v d l,
\end{aligned}
$$

for any $v \in H^{1}(\Omega)$. If $\lambda(\mu) \neq \lambda_{1}$ and we choose $\gamma=\lambda_{1}^{2} \int_{\Gamma_{2}} g S\left(\phi_{1}\right) d l /\left(\lambda(\mu)-\lambda_{1}\right)$ then the last term disappears, and $u$ is indeed a solution to the linear boundary value problem (57), for $\lambda=\lambda(\mu)$. As before it is not very difficult to see that $\lambda(\mu)$ is a continuous function of $0<\mu<\infty$, but this time with

$$
\begin{gathered}
\quad-\infty<\lambda(\mu)<\lambda_{2} \text { for } 0<\mu<\infty, \\
\lambda(\mu) \rightarrow-\infty \text { as } \mu \rightarrow 0 \text { and } \lambda(\mu) \rightarrow \lambda_{2} \text { as } \mu \rightarrow \infty .
\end{gathered}
$$

It is now possible to find $0<\mu_{1}$ such that $\lambda_{1}<\lambda(\mu)<\lambda_{2}$ for $\mu_{1}<\mu<\infty$ and with $\lambda(\mu) \rightarrow \lambda_{1}$ as $\mu \rightarrow \mu_{1}$. The formula (59) thus indirectly provides a variational characterization of the solution to the linear boundary value problem (57) for $\lambda_{1}<\lambda<$ $\lambda_{2}$. For $\lambda=\lambda_{1}$ and $\lambda=\lambda_{2}$ there are, as discussed earlier, no solutions to (57). These results can easily be generalized to give a variational characterization of the solution to the boundary value problem (57) for any interval $\lambda_{n-1}<\lambda<\lambda_{n}$. It would be interesting to see if there were also variational ways to characterize particular solutions to the nonlinear boundary value problem (11) for positive $\lambda$ that are not necessarily small. 
4. Numerical experiments. In this section, we report the results of some numerical experiments for the nonlinear boundary value problem (11) and the associated linear boundary value problem (12). In our computations, we take $\Omega$ to be the region in the first quadrant enclosed by the circular arc $x^{2}+y^{2}=1$ and the four lines $x=0, x=2$, $y=0$, and $y=2$. As indicated in Fig. 4, $\Gamma_{1}$ is taken to be the circular piece of the boundary and $\Gamma_{2}$ consists of the four straight segments labeled $S_{1}, S_{2}, S_{3}$, and $S_{4}$. The Neumann data $g$ is defined as follows: on $S_{1}, S_{3}, S_{4}$, and $\Gamma_{1}, g=0$; on $S_{2}, g$ is the piecewise constant function

$$
g(2, y)= \begin{cases}0 & \text { if } 0 \leq y<\frac{1}{3}-\varepsilon \\ \frac{1}{2 \varepsilon} & \text { if } \frac{1}{3}-\varepsilon \leq y \leq \frac{1}{3}+\varepsilon \\ 0 & \text { if } \frac{1}{3}+\varepsilon<y<\frac{2}{3}-\varepsilon \\ -\frac{1}{2 \varepsilon} & \text { if } \frac{2}{3}-\varepsilon \leq y \leq \frac{2}{3}+\varepsilon \\ 0 & \text { if } \frac{2}{3}+\varepsilon<y \leq 2\end{cases}
$$

This means that $S_{1}, S_{3}$, and $S_{4}$ are insulating, and there is an imposed current pattern on the side $S_{2}$. In the computations, $\varepsilon$ is set to be 0.1 , and the parameter $\alpha$ in the function $F$ (or $f$ ) is set to be 0.5 .

For the linear as well as for the nonlinear boundary value problem, we employed the finite element package PLTMG to compute the numerical solutions and draw the solution diagrams. PLTMG has been developed by R. E. Bank; it is based on piecewise linear triangular elements, and it employs a multigrid strategy for mesh refinement and for the

S3

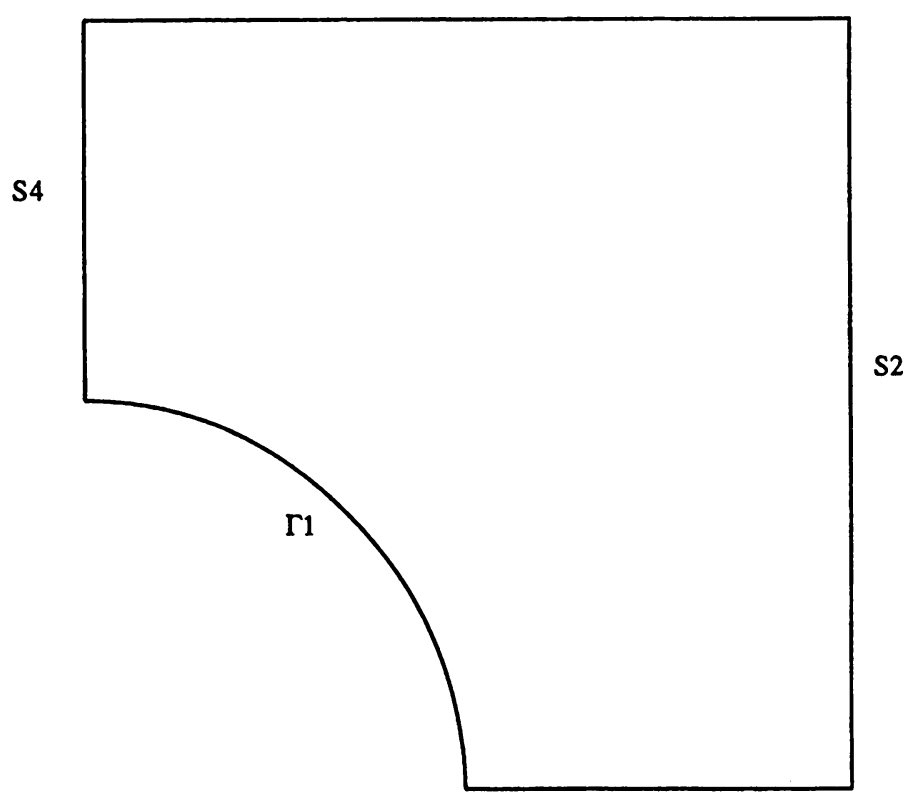




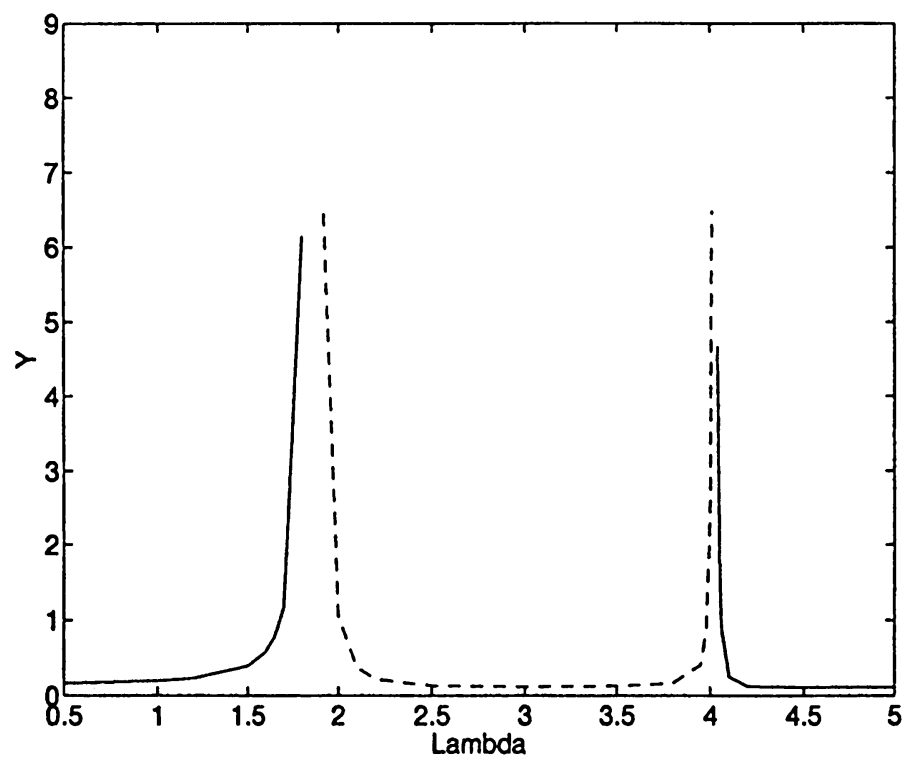

FIG. 5. Solution diagram for the linear problem

solution of the associated finite-dimensional systems. In our computations we used of the order of 1500 degrees of freedom.

The results of our numerical experiments are summarized in Figures 5, 6, and 7. In all figures, the horizontal axis represents the parameter $\lambda$. In Figures 5 and 6 , the vertical axis represents $\left\|U_{\lambda}\right\|_{L^{2}(\Omega)}^{2}$, where $U_{\lambda}$ is a solution to the discrete analog of (12) or (11) respectively. In Fig. 7 , the vertical axis represents $\left\|\nabla U_{\lambda}^{\min }-\nabla U_{\lambda}^{l}\right\|_{L^{2}(\Omega)} /\left\|\nabla U_{\lambda}^{l}\right\|_{L^{2}(\Omega)}$, where $U_{\lambda}^{\min }$ is the discrete solution corresponding to the lower curves in Fig. 6 and $U_{\lambda}^{l}$ is the solution of the discrete analog of the linear boundary value problem.

In Fig. 5 we observe that $\left\|U_{\lambda}\right\|_{L^{2}(\Omega)}^{2}$ blows up when $\lambda$ approaches $\lambda_{1} \approx 1.9$ and $\lambda_{2} \approx 4$. This indicates that the linear boundary value problem does not have solutions corresponding to the parameter values $\lambda_{1}$ and $\lambda_{2}$, because $T\left(\left.u_{0}\right|_{\Gamma_{1}}\right)$ is not orthogonal to the eigenvectors of $T$ associated with $1 / \lambda_{1}$ or those associated with $1 / \lambda_{2}$. Recall that $u_{0}$ is the solution to (57) for $\lambda=0$.

For the nonlinear problem we find three branches of solutions that in a certain sense look like perturbations of the three branches of solutions for the linear problem. We are fairly confident of the accuracy of these branches in a region near the horizontal axis. However, when it comes to the exact crossings of these branches near the vertical axis we expect that they only very crudely approximate the behaviour of the solutions to the continuous problem. We believe that more branches of solutions for the nonlinear problem would show up if we had continued our computations for larger positive $\lambda$ 's. For the linear problem we know that a unique solution exists for all positive $\lambda$, outside a countable set. It might even be that the nonlinear boundary value problem (11) has at least one solution for each $\lambda$. The branch of solutions that starts from $\lambda=0$ has a 


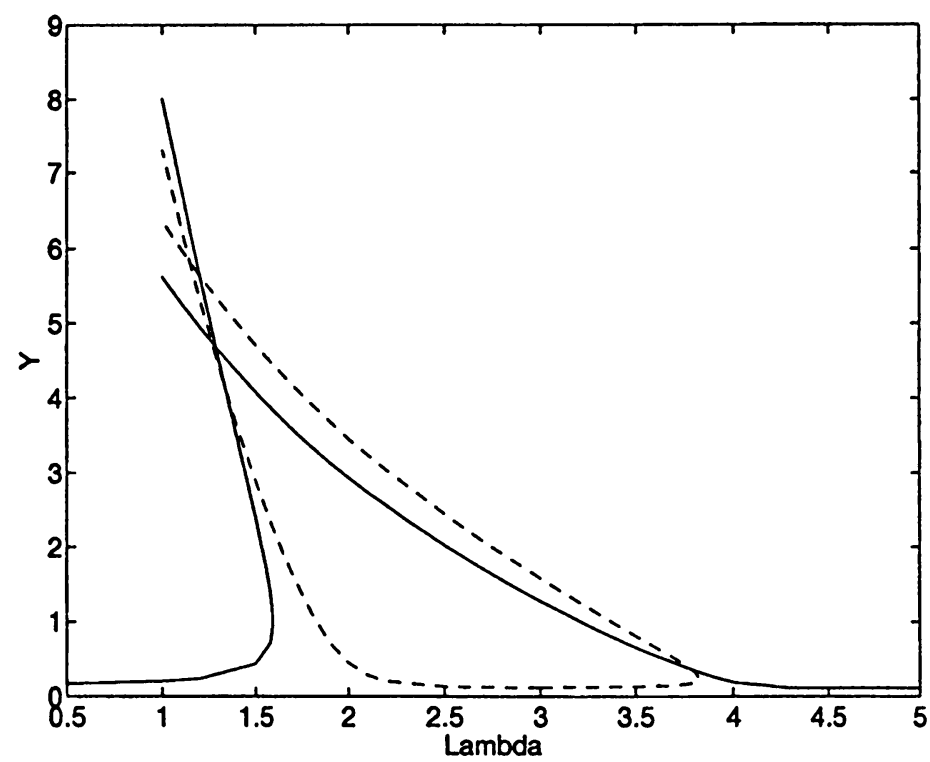

FIG. 6. Solution diagram for the nonlinear problem

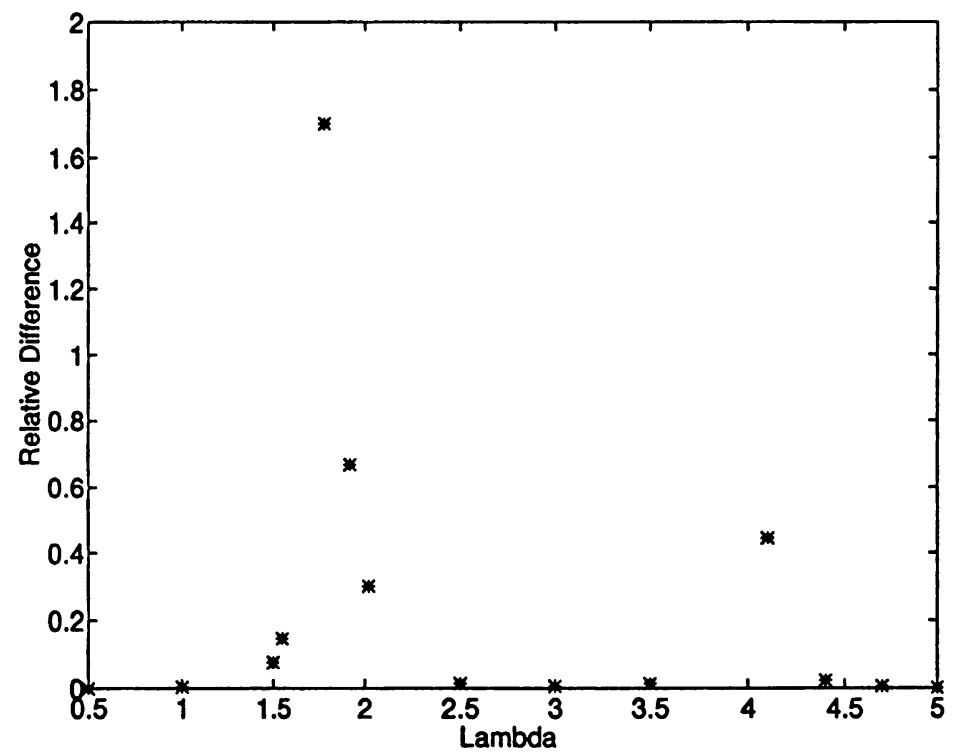

FIG. 7. Relative solution difference 
turning point around $\lambda=1.59$, which is close to $\lambda_{1}$, the first eigenvalue of the linear problem. From Fig. 7 we see that $\left\|\nabla U_{\lambda}^{\text {min }}-\nabla U_{\lambda}^{l}\right\|_{L^{2}(\Omega)} /\left\|\nabla U_{\lambda}^{l}\right\|_{L^{2}(\Omega)}$ is small when $0.5<\lambda<5$ is not close to $\lambda_{1}$ or $\lambda_{2}$.

Acknowledgments. This research was partially supported by the National Science Foundation under grant DMS-9202042 and by the Air Force Office of Scientific Research under contract 89NM605 and URI-RIP grant F49620-93-I-0500.

Appendix. In this appendix we provide a brief introduction to Orlicz spaces, in particular focusing on the way in which the more classical Sobolev spaces are imbedded into these. We refer the interested reader to the book of Adams [1] for an excellent and much more detailed account.

Let $\Phi$ be a real-valued continuous, convex, even function of the real variable $t$, satisfying

$$
\lim _{t \rightarrow 0} \frac{\Phi(t)}{t}=0, \quad \lim _{t \rightarrow \infty} \frac{\Phi(t)}{t}=\infty .
$$

Some examples of such $\phi$ are

$$
\begin{aligned}
& \Phi(t)=|t|^{p}, \quad 1<p<\infty \\
& \Phi(t)=e^{t^{2}}-t^{2}-1 \\
& \Phi(t)=e^{|t|^{p}}-1, \quad 1<p<\infty .
\end{aligned}
$$

Let $\Omega$ be a bounded domain in $\mathbb{R}^{n}$. The Orlicz class $K_{\Phi}(\Omega)$ is the set of all measurable functions, $u$, on $\Omega$ such that

$$
\int_{\Omega} \Phi(u(x)) d x<\infty .
$$

It follows from the convexity of $\Phi$ that $K_{\Phi}(\Omega)$ is a convex set. However, it is not necessarily a vector space. If one does not put additional restrictions on $\Phi$ there are examples where $u \in K_{\Phi}(\Omega)$ and $\lambda \in \mathbb{R}$ but $\lambda u$ is not in $K_{\Phi}(\Omega)$. This is for instance the case with the examples (65) and (66) above. For the first of these examples, $(64), K_{\Phi}(\Omega)$ is clearly a vector space (namely $L^{p}(\Omega)$ ).

In general, the Orlicz space $L_{\Phi}(\Omega)$ is defined as the linear hull of the Orlicz class $K_{\Phi}(\Omega)$, i.e., the smallest vector space containing $K_{\Phi}(\Omega)$. Equipped with the Luxemburg norm

$$
\|u\|_{L_{\Phi}(\Omega)}=\inf \left\{k: \int_{\Omega} \Phi\left(\frac{u}{k}\right) d x \leq 1\right\},
$$

$L_{\Phi}(\Omega)$ becomes a Banach space.

An important theorem, describing the imbedding of the classical integer-order Sobolev spaces, $W_{p}^{m}(\Omega)$, into certain Orlicz spaces, is the following result due to Trudinger, [15], and Adams, [1]. In a sense this theorem can be viewed as a limiting case of the more well-known Sobolev Imbedding Theorem. 
Theorem. Let $\Omega$ be a bounded domain in $\mathbb{R}^{n}$ having the cone property. Let $m<n$ be a positive integer and set $p=n / m>1$. Define $\Phi$ to be the function

$$
\Phi(t)=e^{|t|^{p /(p-1)}}-1 \text {. }
$$

Then one has the continuous imbedding

$$
W_{p}^{m}(\Omega) \rightarrow L_{\Phi}(\Omega)
$$

REMARK. A similar result holds for fractional-order Sobolev spaces. Such a result is for instance found in Theorem 9.1 of [13] with a minor difference being that it is formulated with a base domain that is all of $\mathbb{R}^{n}$. We are specifically interested in the case $p=2, n=1$ and $m=\frac{1}{2}$ (and $r=2$ ), in which case this theorem asserts that one has the continuous imbedding $W_{2}^{1 / 2,2}(\mathbb{R}) \rightarrow L_{\widetilde{\Phi}}(\mathbb{R})$ with

$$
\widetilde{\Phi}(t)=\sum_{j \geq 2} \frac{t^{2 j}}{j !}=e^{t^{2}}-t^{2}-1 .
$$

The space $W_{2}^{1 / 2,2}$ is a so-called Besov space, but as established in Theorem 6.4.4 of [3] this particular one coincides with the classical Sobolev space $H^{1 / 2}$. Since the space $H^{1 / 2}$ is also contained in $L^{2}$ it now follows immediately that one has the continuous imbedding $H^{1 / 2}(\mathbb{R}) \rightarrow L_{\Phi}(\mathbb{R})$ with

$$
\Phi(t)=e^{t^{2}}-1
$$

Composition with natural extension and restriction operators now leads to the validity of the continuous imbedding $H^{1 / 2}(I) \rightarrow L_{\Phi}(I)$ for any bounded, open interval $I \subset \mathbb{R}$. Since the (one-dimensional) boundary part $\Gamma_{1}$ is smoothly isomorphic to a bounded interval it follows immediately that one has the imbedding $H^{1 / 2}\left(\Gamma_{1}\right) \rightarrow L_{\Phi}\left(\Gamma_{1}\right)$. It is the validity of this imbedding that we have used on several occasions in this paper.

\section{REFERENCES}

[1] R. A. Adams, Sobolev Spaces, Academic Press, New York, 1975

[2] R. Baboian, Corrosion-A Problem of International Importance, Corrosion Testing and Evaluation: Silver Anniversary Volume, ASTM STP 1000, R. Baboian and S. S. Dean, eds., American Society for Testing and Materials, Philadelphia, 1990, pp. 7-13.

[3] J. Bergh and J. Löfström, Interpolation Spaces, Springer-Verlag, Berlin, 1976.

[4] H. Brezis and L. Nirenberg, book in preparation

[5] J. Deconinck, Current Distributions and Electrode Shape Changes in Electrochemical Systems, Lecture Notes in Engineering, Vol. 75, Springer-Verlag, Berlin, 1992

[6] Z.-C. Han, Prescribing Gaussian curvature on $S^{2}$, Duke Mathematical Journal 61, 679-703 (1990)

[7] L. S. Hou and J. C. Turner, Analysis and finite element approximation of an optimal control problem in electrochemistry with current density controls, Numer. Math. 71, 289-315 (1995)

[8] M. A. Krasnosel'skii and Ya. B. Rutickii, Convex Functions and Orlicz Spaces, Noordhoff, Groningen, 1961

[9] D. Landolt, Electrochemical and materials science aspects of alloy deposition, Electrochimica Acta 39, 1075-1090 (1994)

[10] M. Matlosz, C. Creton, C. Clerc, and D. Landolt, Secondary current distribution in a Hull cell, J. Electrochem. Soc. 134, 3015-3021 (1987)

[11] J. Moser, On a nonlinear problem in differential geometry, In Dynamical Systems, M. Peixoto, Editor, Academic Press, New York, 1973

[12] J. S. Newman, Electrochemical Systems, Prentice-Hall, Englewood Cliffs, NJ, 1973 
[13] J. Peetre, Espaces d'Interpolation et Théorème de Soboleff, Ann. Inst. Fourier (Grenoble) 16, 279317 (1966)

[14] P. Ponthiaux, F. Wenger, and J. Galland, Study of the anodic current-voltage curve of an iron-nickel alloy in normal sulfuric acid, J. Electrochem. Soc. 142, 2204-2210 (1966)

[15] N. S. Trudinger, On imbeddings into Orlicz spaces and some applications, J. Math. Mech. 17, 473-483 (1967)

[16] H. H. Uhlig and R. W. Revie, Corrosion and Corrosion Control, John Wiley and Sons, New York, 1985

[17] N. G. Zamani, J. F. Porter, and A. A. Mufti, A survey of computational efforts in the field of corrosion engineering, International Journal for Numerical Methods in Engineering 23, 1295-1311 (1986) 